\title{
Water fluoridation and Down's syndrome evidence inconclusive
}

\author{
Is there an association between water fluoridation and Down's syndrome?
}

\section{Whiting P, McDonagh M Kleijnen J. Association of Down's syndrome and water fluoride level: a systematic review of the evidence. BMC Public Health 2001; 1:6 \\ [www.biomedcentral.com/1471-2458/1/6}

Data sources A qualified librarian searched 25 specialist databases, including Medline, Embase, Toxline and the Current Contents Science Citation Index, from database inception to February 2000. In addition, searches of Index Medicus (1945-1959) and Excerpta Medica (19551973) were undertaken by hand. Additional references were sought from individuals and organisations through a dedicated website for this review [www.york.ac.uk/inst/crd/fluoride.htm], and through members of a specifically designated advisory panel. Published and unpublished studies in any language were included.

Study selection All study designs were included that compared the incidence of Down's syndrome in populations where different levels of fluoride occurred in water supplies, either naturally or added artificially. Data extraction and synthesis Two reviewers independently assessed each paper for inclusion, and disagreements were resolved through consensus. A qualitative analysis was conducted.

Results The six studies included were all ecological in design and had poor validity scores. The estimates of the crude relative risk ranged from 0.84 to 3.0. Four studies showed no significant association between the incidence of Down's syndrome and water fluoride level whereas two studies by the same author found a significant $(P<0.05)$ positive association (increased Down's syndrome incidence with increased water fluoride level). Only two of the studies controlled for confounding factors and only one of these presented summary outcome measures.

Conclusions The evidence of an association between water fluoride level and Down's syndrome incidence is inconclusive. The quality of the studies included in the review was relatively low, however, and further high quality research is needed.

\section{Commentary}

The UK Department of Health commissioned a review of the safety and efficacy of drinking water fluoridation, including an investigation of whether the evidence supported a beneficial effect of water fluoridation and whether there was any evidence of adverse effects. ${ }^{1}$ Other than for dental fluorosis, bone fracture and cancer, there was little evidence available on adverse effects in humans. ${ }^{1,2}$ Down's syndrome was the most discussed of the other adverse effects reported, and was therefore selected as the focus for the paper summarised here.

Regarding Down's syndrome, exposure to risk factors should be measured at the time at which the abnormality may occur, ie, around the time of conception. The main risk factor is maternal age. Possible confounding factors include race, radiation, increased parity, and season, with a peak in summer. Quality investigations would make attempts to control for these confounding factors. As

Address for correspondence: Penny Whiting, Centre for Reviews and Dissemination, University of York, York Y010 5DD, UK. E-mail: pfw2@york.ac.uk. mentioned above, fluoride level has been suggested as a risk factor, and has been investigated by a number of studies.

The studies selected did not provide sufficient information to permit pooling of data or investigation of statistical heterogeneity. Insufficient data were available to investigate publication bias using standard methods.

This review suggests that the evidence for an association between water fluoride level and the incidence of Down's syndrome is weak. All the identified studies were of poor quality. None had a prospective follow-up, incorporated any form of blinding, had a baseline survey or stated how the level of fluoride in the water was calculated. Controlling for confounding factors was generally inadequate. All six studies measured population rather than individual exposure to fluoridated water and therefore are particularly susceptible to confounding. None of the studies controlled for exposure to other sources of fluoride, such as toothpaste or tablets.

All the studies identified were conducted at least 20 years ago so there may be a problem in generalising results to the present time. Factors such as the mother's period of time of exposure to fluoridation, changing demographics of maternal age at birth and abortion rate related to screening for Down's syndrome may affect this ability to generalise the results.

Since the quality of the studies included in this review was relatively low, further high-quality research is still needed. Future studies should measure individual exposure to water fluoride and control appropriately for confounding factors. Study areas should be chosen at random and investigators should be blinded to the fluoridation status of mothers. The population denominator selected to measure the risk of a Down's syndrome birth should relate to the total number of births, not to the overall population of the study area. Case ascertainment should be as complete as possible and should be identical in all population studies.

\section{Practice point}

- The practitioner should be aware of any possible adverse effects of fluoridation but there is no conclusive evidence of association between water fluoridation and Down's syndrome.

\section{Richard D Bebermeyer}

Department of Restorative Dentistry and Biomaterials, University of Texas Health Science Center at Houston - Dental Branch, Houston, TX 77030, USA

1. National Health Service Centre for Reviews and Dissemination. A systematic review of public water fluoridation. York: University of York; 2000; 3:67. dbchISBN 1 90064016.

2. MMWR Morbidity and Mortality Weekly Report. Recommendations for using fluoride to prevent and control dental caries in the United States. 2001; 50(RR-14):25.

Evidence-Based Dentistry (2004) 5, 39. doi:10.1038/sj.ebd.6400251 